Improved dead-time correction for PET scanners: application to small-animal PET

This article has been downloaded from IOPscience. Please scroll down to see the full text article.

2013 Phys. Med. Biol. 582059

(http://iopscience.iop.org/0031-9155/58/7/2059)

View the table of contents for this issue, or go to the journal homepage for more

Download details:

IP Address: 157.193.1.253

The article was downloaded on 24/06/2013 at 22:53

Please note that terms and conditions apply. 


\title{
Improved dead-time correction for PET scanners: application to small-animal PET
}

\author{
E Vicente ${ }^{1,2}, \mathbf{J}$ L Herraiz ${ }^{3}$, S España $^{4}$, E Herranz $^{1}$, M Desco $^{5,6}$, \\ $\mathbf{J} \mathbf{J}$ Vaquero ${ }^{6}$ and $\mathbf{J}$ M Udías ${ }^{1}$ \\ ${ }^{1}$ Grupo de Física Nuclear, Departamento de Física Atómica, Molecular y Nuclear, \\ Universidad Complutense de Madrid, CEI Moncloa, Spain \\ ${ }^{2}$ Instituto de Estructura de la Materia, Consejo Superior de Investigaciones Científicas (CSIC), \\ Madrid, Spain \\ ${ }^{3}$ Madrid-MIT M+Visión Consortium, Research Laboratory of Electronics, \\ Massachusetts Institute of Technology, Cambridge, MA, USA \\ ${ }^{4}$ Medical Image and Signal Processing Group, Department of Electronics and Information \\ Systems, Ghent University Hospital, Belgium \\ ${ }^{5}$ Unidad de Medicina y Cirugía Experimental, Hospital General Universitario Gregorio \\ Marañón, CIBERSAM, Madrid, Spain \\ ${ }^{6}$ Departamento de Bioingeniería e Ingeniería Aeroespacial, Universidad Carlos III de Madrid, \\ Spain \\ E-mail: esther@nuclear.fis.ucm.es
}

Received 19 November 2012, in final form 5 February 2013

Published 4 March 2013

Online at stacks.iop.org/PMB/58/2059

\begin{abstract}
Pile-up and dead-time are two main causes of nonlinearity in the response of a PET scanner as a function of activity in the field of view (FOV). For a given scanner and acquisition system, pile-up effects depend on the material and size of the object being imaged and on the distribution of activity inside and outside the FOV, because these factors change the singles-to-coincidences ratio (SCR). Thus, it is difficult to devise an accurate correction that would be valid for any acquisition. In this work, we demonstrate a linear relationship between SCR and effective dead-time, which measures the effects of both dead-time (losses) and pile-up (gains and losses). This relationship allows us to propose a simple method to accurately estimate dead-time and pile-up corrections using only two calibration acquisitions with, respectively, a high and low SCR. The method has been tested with simulations and experimental data for two different scanner geometries: a scanner with large area detectors and no pile-up rejection, and a scanner composed of two full rings of smaller detectors. Our results show that the SCR correction method is accurate within $7 \%$, even for high activities in the FOV, and avoids the bias of the standard single-parameter method.
\end{abstract}

(Some figures may appear in colour only in the online journal) 


\section{Introduction}

The count rate of a PET scanner as a function of activity in the field of view (FOV) has nonlinear contributions due to dead-time, pile-up and random coincidences (Knoll 2000, Cherry et al 2003). An adequate estimation of these nonlinear effects is essential for accurate quantitative studies (Bendriem and Townsend 1998). Several methods have been proposed to measure or estimate and correct for random coincidences (Knoll 2000, Williams et al 1979, Casey and Hoffman 1986, Badawi et al 1999, Stearns et al 2003, Brasse et al 2005). In this work, we focus on the simultaneous study of pile-up and dead-time effects, which we call 'effective dead-time', as they are closely related. Dead-time is a measurement of the scanner's inability to process every valid physical event because the acquisition system is busy processing previous events and may depend on many components such as detectors, electronics, hard disk or software. Pile-up denotes the situation in which additional photons deposit energy in either a whole or a part of the detector while the data acquisition channel is still processing (integrating) the energy from a previous event (Knoll 2000). Under these conditions, the energy from these photons is added to that from the photon which triggered the acquisition, as if they formed a single event. Pile-up effects are more frequent at high count rates and they become more significant when large area detectors are read by the same data acquisition channel (Mankoff et al 1989, Smith et al 1994, Vicente et al 2007, 2008). When pile-up causes loss of counts, it can be considered one of the components of a generalized dead-time concept. As such, dead-time not related to pile-up and loss of counts due to pile-up are considered together as dead-time (Yu and Fessler 2000, Hoffman et al 1983, Germano and Hoffman 1991). Nevertheless, it is noteworthy that pile-up events can lead to both loss and gain of counts.

Pile-up induces different kinds of errors in PET data (Furrer et al 1984). If the pile-up photon contributes a signal large enough to make an otherwise valid (within the given energy window) event fall outside the upper level discriminator (ULD), the event will be rejected, thus contributing to system dead-time. Conversely, pile-up can also shift low-energy events inside the energy window, making them appear valid. This pile-up action, which increases counts during acquisition, could be considered a source of negative dead-time. In addition, pile-up can also shift the detected energy of photons that would lie within the energy window into an apparent value of energy which would still lie inside the energy window. In this case, pile-up would not change the number of counts but it would lead to mispositioned coincidences (as in the case of pile-up gains), unless piled-up events are identified and rejected (Germano and Hoffman 1990, Badawi and Marsden 1999).

Pile-up effects can be reduced in several ways at the hardware level (Smith et al 1994). For instance, the likelihood of pile-up can be reduced by using the shortest possible integration time. However, integration time cannot be arbitrarily short, because enough light has to be integrated to ensure acceptable spatial and energy resolutions. For this reason, short integration times demand scintillators with very short light decay times. Other approaches to alleviating the effect of pile-up on the count rate ability of the scanner have been proposed by Germano and Hoffman (1991), Sjöland and Kristiansson (1994) and Imperiale and Imperiale (2001). Although all of these pile-up identification and rejection mechanisms can prove effective at reducing the spectral distortions associated with pile-up, count losses must still be corrected if an accurate quantitative analysis is pursued.

Several methods have been proposed for characterizing count rate losses due to pile-up and dead-time. Hoffman et al (1983) calculated dead-time corrections from on-line measurements of triple coincidence events. Stearns et al (1985) measured dead-time losses independently for both single and coincidence events and combined them to obtain a total dead-time measurement. Mazoyer et al (1985) proposed a scheme that corrected both emission and 
transmission data based on a paralyzing dead-time model using the total coincidence rate. Yamamoto et al (1986) proposed a method based on a nonlinear function of the random coincidences measured off-line. They showed that, analogous to random counts, the deadtime fraction depends on the object size. The model of Daube-Witherspoon and Carson (1991) for emission and transmission in 2D PET scanners takes into account coincidence losses due to singles losses and multiple events, as well as mispositioning errors at higher count rates caused by pulse pile-up. Eriksson et al (1994) assumed that data loss effects can be factorized into two components, one derived from the detector processing system (identifying all single events) and another associated with the data processing system.

In most of these proposals, the correction for count losses due to dead-time and pileup is derived from a combination of analytical models of data acquisition electronics and empirical data from phantom scans at various count rates. Nevertheless, to the best of our knowledge, no simple protocols (i.e. those not requiring additional hardware) accurately estimate and take into account pile-up effects in a general acquisition. This is because pile-up effects depend on the singles-to-coincidences ratio (SCR), which is in turn determined by the size and material of the object placed in the FOV, as well as by the source distribution (inside/outside the FOV). This means that dead-time or pile-up corrections estimated from the acquisition of a given phantom would only be approximate if applied to a different object. Using this kind of simplified correction, quantification of images with significant pile-up and dead-time effects becomes compromised. More sophisticated methods can better estimate the corrections, but they usually rely on additional information obtained during acquisition. For instance, Yamamoto et al (1986) obtained improved estimates of deadtime and pile-up effects by measuring off-line random coincidences, which is not always achievable.

In this work, we propose a simple method to identify the effective dead-time $(\tau)$ which restores the correct linear relationship between activity in the FOV and count rate measured by the scanner. This effective dead-time can be estimated for any acquisition, takes into account both pile-up losses and gains as well as dead-time losses, and does not require special hardware, processing of the events or recording of information beyond that which is commonly available in PET systems. The method requires only two calibration acquisitions and is based on the linear relationship between $\tau$ and SCR. We verified that the method performs well in a scanner for which pile-up effects are particularly important, as is the case of the rPET scanner (Vaquero et al 2005), with large area detectors and no electronic pile-up rejection. In order to support the applicability of the method, we present detailed results for Sedecal rPET and for the Sedecal Argus scanner (formerly GE eXplore Vista) (Wang et al 2006b).

\section{Materials and methods}

\subsection{The decaying source method to estimate effective dead-time}

Dead-time behaviour in a PET scanner as a function of the count rate is often estimated using a decaying source experiment (Knoll 2000). Repeated measurements of total coincidence rates are obtained for different activities of a decaying source. In the limit of very low activities, when dead-time (and other nonlinear effects) vanishes, the measured count rate, $m(t)$, would coincide with the true incident count rate $n(t)$. Since the true incident count rate for larger activities can be easily calculated according to the decay time correction, the ratio of incident to measured counts $\left([m(t)]_{d} /[n(t)]_{d}\right.$, where the subscript $d$ stands for decay corrected) can be estimated for any activity, thus making it possible to empirically obtain a dead-time correction for a given acquisition and scanner. The dead-time correction factor $[m(t)]_{d} /[n(t)]_{d}$ can be fitted to an 
Table 1. Description of the simulated phantoms. PS: point source; LS: line source; SC: small cylinder; LC: large cylinder; $d$ : cylinder diameter in $\mathrm{cm}(d=[0.4,1,2,3,4,5]) ; \phi$ : cylinder diameter; $h$ : cylinder height.

\begin{tabular}{|c|c|c|c|c|}
\hline \multirow[b]{2}{*}{ Phantom name } & \multicolumn{2}{|c|}{ Description $^{\mathrm{a}}$} & \multicolumn{2}{|c|}{ Dimensions } \\
\hline & Source (isotope: ${ }^{18} \mathrm{~F}$ ) & Object & Source $\phi / h(\mathrm{~cm})$ & Object $\phi / h(\mathrm{~cm})$ \\
\hline$P S$ & Point source & Water cubic box & $0.05 /-$ & $1 \times 1 \times 1$ \\
\hline $\begin{array}{l}\text { Water }(S C) \\
\text { Al }(S C)\end{array}$ & Small cylinder (SC) & $\begin{array}{l}\text { Small water cylinder } \\
\text { Small aluminium } \\
\text { cylinder }\end{array}$ & $0.9 / 6.5$ & $0.9 / 6.5$ \\
\hline$G S O(S C)$ & & Small GSO cylinder & & \\
\hline$P b(S C)$ & & Small lead cylinder & & \\
\hline Water $(L C)$ & Large cylinder (LC) & Large water cylinder & $5.5 / 5.0$ & $5.5 / 5.0$ \\
\hline $\begin{array}{l}\text { Water }(L S \text { and } C d) \\
d=[0.4,1,2,3,4,5]\end{array}$ & Line source (LS) & Water cylinder & $0.2 / 0.4$ & $d / 4.0$ \\
\hline $\begin{array}{l}A l(L S \text { and } C d) \\
d=[0.4,1,2,3,4,5]\end{array}$ & & Aluminium cylinder & $d / 4.0$ & \\
\hline
\end{tabular}

a All phantoms (sources and objects) are placed at the centre of the FOV.

analytic expression containing an effective dead-time $\tau$, using paralyzable, non-paralyzable or mixed models (Knoll 2000).

Sometimes dead-time corrections for a given scanner are obtained using a reference acquisition of an intermediate-size phantom (Knoll 2000, Germano and Hoffman 1988). Once the effective dead-time $\tau$ is obtained from this phantom, corrections evaluated with the same value of $\tau$ are applied to all acquisitions performed by the same scanner. Hereinafter, we refer to this method as the 'single-parameter method'.

\subsection{New method to estimate effective dead-times: the SCR method}

Yamamoto et al (1986) and Thompson and Meyer (1987) studied the dependence of $\tau$ on the size of the object in the FOV. We performed a more detailed study using simulations of decaying sources with different phantom sizes and materials and different energy windows for the acquisition. Table 1 summarizes the simulated phantoms used, as well as the point and capillary sources embedded in phantoms of different materials and sizes. The effective deadtime for each phantom acquisition was obtained with the decaying source method explained in the previous section. We expected these effective dead-time values $\tau$ to depend on the SCR for each acquisition. Thus, we plotted effective dead-times against the true SCR values for each acquisition. True SCR values are free from dead-time, pile-up and random count effects and can easily be obtained from a decaying source experiment at the limit of vanishing activity.

It can be shown that a linear relationship exists between the effective dead-time, $\tau$, and SCR. For the sake of simplicity, we will show the derivation in the limit of low rates and later we will analyse its validity for arbitrary rates.

The fraction of coincidences that are free of pile-up and dead-time, $f_{c}$, can be expressed as (Knoll 2000)

$$
f_{c} \cong 1-n \tau,
$$

where $n$ is the detected coincidence rate free of dead-time (and pile-up) and $\tau$ the effective dead-time for coincidences. We can define a dead-time component (possibly including pile-up effects) that affects every single event recorded by the scanner, represented by a 'singles' dead-time $\tau_{S}$. Furthermore, another dead-time component is derived from the processing of 
coincidences, represented by a 'coincidence-only' dead-time $\tau_{C}$. In the limit of low rates, the fraction of coincidences which remain free of dead-time (and pile-up) is related to the fraction of singles free of dead-time (and pile-up), $1-n_{S} \tau_{S}$, as follows:

$$
f_{c}=1-n \tau=\left(1-n_{S} \tau_{S}\right)^{2} \cdot\left(1-n \tau_{C}\right),
$$

where $n_{S}$ is the detected singles rate free of dead-time (and pile-up). This equation shows that, in order to record a coincidence, both single events must survive the singles dead-time, and the resulting coincidence must survive the coincidence-only dead-time. From equation (2), we can obtain $\tau$, disregarding the terms of second order and higher in $\tau_{C}$ and $\tau_{S}$ :

$$
\tau=2 \cdot \tau_{S} \cdot \frac{n_{S}}{n}+\tau_{C}
$$

Using SCR $=n_{S} / n$, equation (3) becomes

$$
\tau=2 \cdot \tau_{S} \cdot \operatorname{SCR}+\tau_{C} .
$$

Equation (4) shows a linear relationship between the effective dead-time observed in the acquired coincidences and SCR. It also shows that the slope and intercept of a linear fit of the effective $\tau$ versus SCR will make it possible to estimate the effective singles dead-time (including pile-up effects) and the coincidences-only dead-time, respectively.

Equation (4) is derived here for low count rates $\left(n_{S} \tau_{S} \ll 1\right.$ and $\left.n \tau_{C} \ll 1\right)$ and, within this constraint, it is valid for both the paralyzable and non-paralyzable models. Furthermore, this equation is exactly valid for arbitrary count rates for paralyzable dead-time systems, for which the fraction of coincidences that are free of pile-up and dead-time, $f_{c}$, is given by $\mathrm{e}^{-n \tau}$. For non-paralyzable systems, the expression valid for any rate is

$$
\tau=2 \cdot \tau_{S} \cdot \operatorname{SCR}\left(1+\frac{n_{S} \tau_{S}}{2}\right)+\tau_{C}\left(1+n_{S} \tau_{S}\right)^{2},
$$

where the linear relationship with SCR is modified by $n_{S} \tau_{S}$ terms. It is reasonable to assume that many realistic situations would be described by a combination of paralyzable and nonparalyzable models and thus equation (4) is expected to be valid even for high coincidence rates. Indeed equation (4) should be valid provided $n_{S} \tau_{S}$ is small.

To use equation (4) the scanner must have access to singles rates in order to estimate SCR values. Singles rates can be estimated with relatively simple trigger counters in each detector, which can be implemented with very small dead-time $\tau_{S}$. The singles rate free of dead-time (and pile-up), $n_{S}$, can be employed to obtain the SCR even though the coincidence rate is computed within an energy window. Indeed this asymmetric way of computing the SCR, without applying energy window to the singles and measuring the coincidences within an energy window, is compatible with the derivation of equation (4). The small value of $\tau_{S}$ that can be achieved with simple circuits, which just counts trigger events, facilitates the use of equation (4) even when relatively high singles rates are involved.

One drawback of this approach appears in the case of acquisitions for which the SCR changes significantly during the acquisition, such as dynamic studies. For these cases, it would be preferable to work with the actual count rates $m_{S}$ and $m\left(\mathrm{SCR}_{m}=m_{S} / m\right)$, which would include dead-time (and pile-up) effects. In these cases, equation (4) would still be valid in the limit of low rates with a simple replacement of $n$ by $m$.

Based on equation (4), we propose to characterize the combined effect of pile-up and deadtime for a given scanner using the parameters $\tau_{C}$ and $\tau_{S}$, which can be estimated from two effective dead-times $\tau_{1}$ and $\tau_{2}$ (see figure 1) obtained from two decaying source experiments, one with high SCR and one with low $\mathrm{SCR}^{7}$. The specific effective dead-time, $\tau$, needed to correct another acquisition, can be obtained from equation (4). Other than assuming that the

7 No energy window was applied to measure the singles rates. 


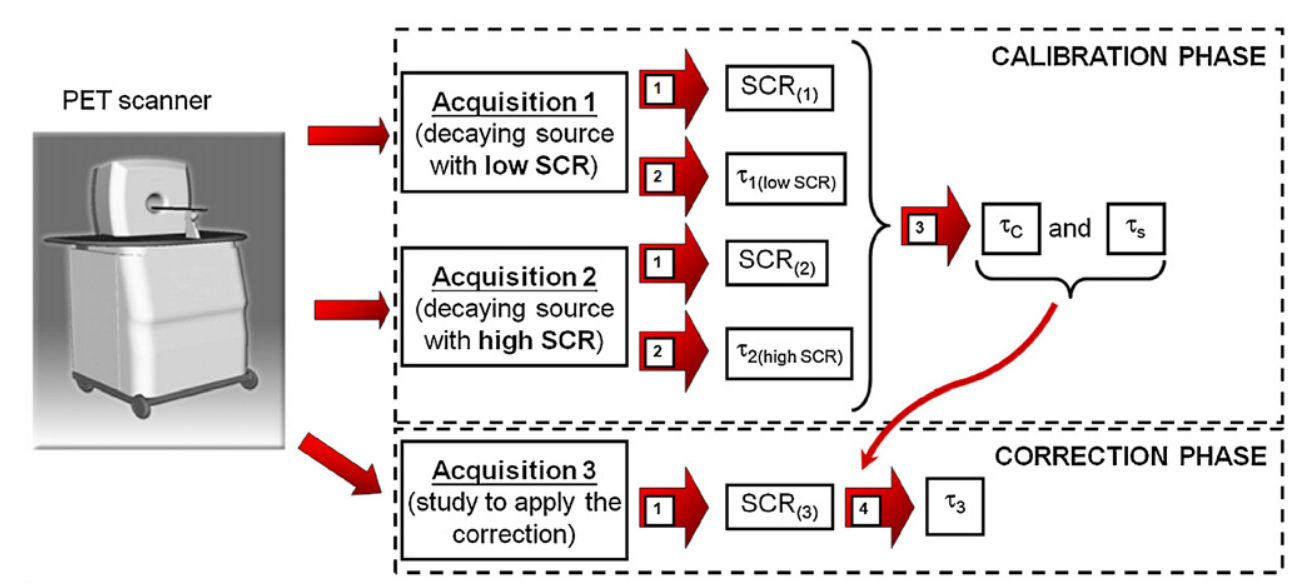

1 SCR computed from the singles rate (no Energy Window (EW) restriction) and the coincidences rate (in a specific EW).
2 Linear fit to obtain $\tau$ using an analytic model (paralyzable, non paralyzable or mixed).
3 Linear fit to obtain the parameters of equation (4).
4 Using the relationship (4) with the parameters obtained in the calibration step and the SCR of the acquisition, the specific $\tau_{3}$ is obtained.

Figure 1. Flowchart of the SCR method to obtain the effective dead-time correction.

scanner can yield reasonably accurate estimates of the SCR for each acquisition, neither further hardware nor additional count processing is required. We call this procedure the 'SCR method' (figure 1).

\subsection{Validation of the SCR method}

In this work we used two small-animal PET scanners with different geometries and features.

Sedecal rPET. This scanner (SEDECAL Medical Systems) has four large detectors arranged in two orthogonal pairs which rotate $180^{\circ}$ (Vaquero et al 2005). Each detector comprises a single-layer array of $28 \times 28$ MLS crystals $\left(1.5 \times 1.5 \times 12 \mathrm{~mm}^{3}\right)$ optically coupled to a Hamamatsu H8500 flat-panel PS-PMT (size: $52 \times 52 \times 28 \mathrm{~mm}^{3}$; effective area: $49 \times 49 \mathrm{~mm}^{2} ; 8 \times 8$ Anode Pixels' matrix). Since each PS-PMT is read by the same data acquisition subsystem, the whole area of the detector is subject to pile-up effects. The scanner has a ring diameter of $160 \mathrm{~mm}$ with effective transverse and axial FOVs of $44.8 \mathrm{~mm}$.

Sedecal Argus (formerly GE eXplore Vista). The Sedecal Argus, formerly marketed by GE Healthcare as GE eXplore Vista (Wang et al 2006b), uses a block detector design with a dual-layer phoswich crystal array coupled to a Hamamatsu R8520-C12 PS-PMT. The front crystal layer is a $13 \times 13$ array of $1.45 \times 1.45 \times 7 \mathrm{~mm}^{3}$ LYSO crystals, and the back layer is a $13 \times 13$ array of $1.45 \times 1.45 \times 8 \mathrm{~mm}^{3}$ GSO crystals. The detectors are arranged into two rings of 18 detectors per ring with a ring diameter of $118 \mathrm{~mm}$ and transaxial FOV of $68 \mathrm{~mm}$. The axial coverage of this system is $48 \mathrm{~mm}$.

Both, rPET and Argus scanners, have the capability to estimate $n_{S}$.

In order to evaluate the method proposed to correct for dead-time and pile-up effects (SCR method), we first analysed the decaying source method using both experimental data and simulations for the Water (SC) (small cylinder) phantom acquired with the rPET scanner. We assumed a non-paralyzable behaviour, although similar effective dead-time values $(\tau)$ were achieved with paralyzable expressions (differences around 1-2\%) provided the values of the dead-time correction factor are not extreme, as is the case of the scanners we analysed 


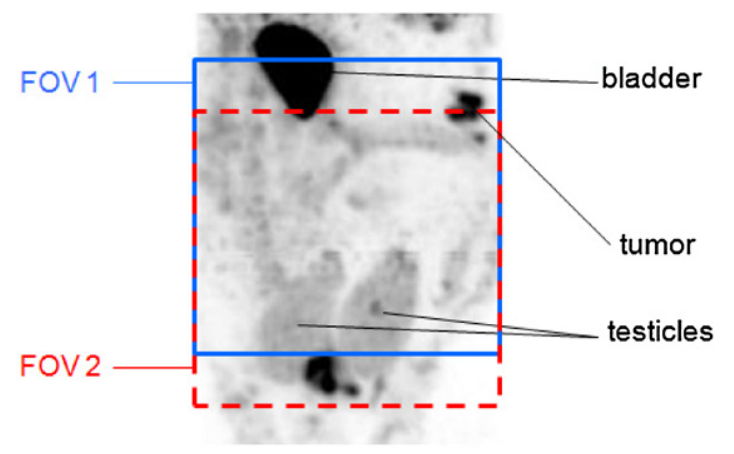

Figure 2. Bed positions used in the simulation of a voxelized image of a rat. 'FOV 1' refers to the rat with the hot bladder at the edge of the FOV while 'FOV 2' has the hot bladder outside the FOV, but very close to its edge.

under their usual operational ranges. We performed a decaying source experiment with the rPET system using a $S C$ phantom $\left(0.9 \mathrm{~cm}\right.$ in diameter, $6.5 \mathrm{~cm}$ in length) filled with ${ }^{18} \mathrm{~F}$ in water ('Water (SC)', see table 1) placed at the centre of the FOV of the scanner. For reference, and although not needed in the procedure, the initial activity was measured in a well counter with an accuracy of $\pm 5 \%$. Several consecutive acquisitions of 5 min with an energy window of 400-700 keV were obtained, starting from an initial activity of $900 \mu \mathrm{Ci}$ (activity concentration of $254.6 \mu \mathrm{Ci} \mathrm{cc}{ }^{-1}$ ) and a total count rate of $130 \mathrm{kcps}$. In addition, acquisitions of this source were simulated with PeneloPET (España et al 2009) to mimic the experimental setup. In the simulations, we used a coincidence dead-time of $1.6 \mu \mathrm{s}$, which includes the conversion time of the analogue-to-digital converter (ADC) and an integration time of $0.22 \mu \mathrm{s}$, both of which values suit rPET hardware. From the simulations, it is possible to estimate the individual contributions to the total effective dead-time that can be split into three components: strict dead-time $\left(\tau_{\mathrm{DT}}\right)$, pile-up losses $\left(\tau_{p \text { (loss) }}\right)$ and pile-up gains ( $\left.\tau_{p(\text { gain })}\right)$, according to $\tau=\tau_{\mathrm{DT}}+\tau_{p \text { (loss) }}-\tau_{p \text { (gain) }}$.

The simulated phantoms described in table 1 were used to study and validate the SCR method. The effective dead-time for each phantom acquisition was estimated with the decaying source method, as explained in section 2.1. The SCR method explained in section 2.2 was applied using the $P S$ and the Water $(L C)$ phantoms as the two calibration experiments for both scanners described above. Random coincidences in the Argus scanner were removed from the acquisition prior to the data analysis to compute $\tau$. This was not necessary for the rPET scanner, as the fraction of random counts for this scanner was negligible (see figure 4).

Further simulations were performed in order to verify the SCR method for asymmetric activity distributions in the FOV. Using a voxelized image of a rat as the source input in the simulation, we chose two different situations (bed positions, see figure 2). The image was derived from a reconstruction of an experimental acquisition analysed in Vicente et al (2007). In the first case, we simulated the bed position of the rat with a hot bladder at the edge of the FOV ('FOV 1' in the figure); in the second case the hot bladder was outside, but very close to the edge of the FOV ('FOV 2' in the figure). The activity ratio of the hot bladder to the testicles was $45: 1$. These simulations were used to compare the $\tau$ value obtained from the decaying source method and the one obtained using the SCR method.

We also estimated the error introduced when the effective dead-time obtained for a particular phantom is used to correct dead-time for another phantom with a different SCR, that is, when the single-parameter method is used. We compared the results with the ones obtained correcting with effective dead-times estimated with the SCR method. 
(A)

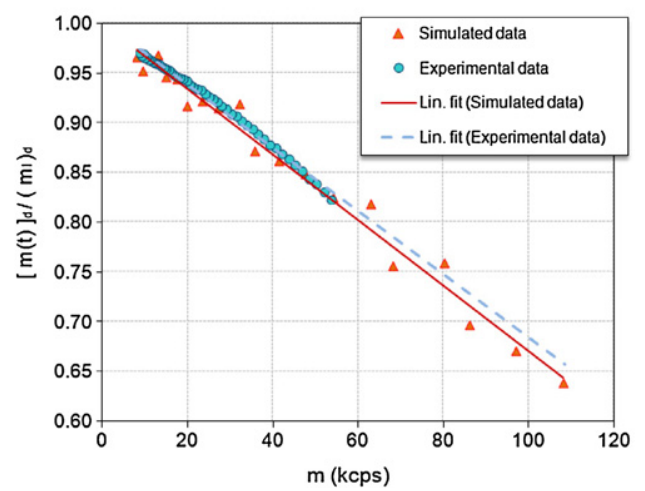

(B)

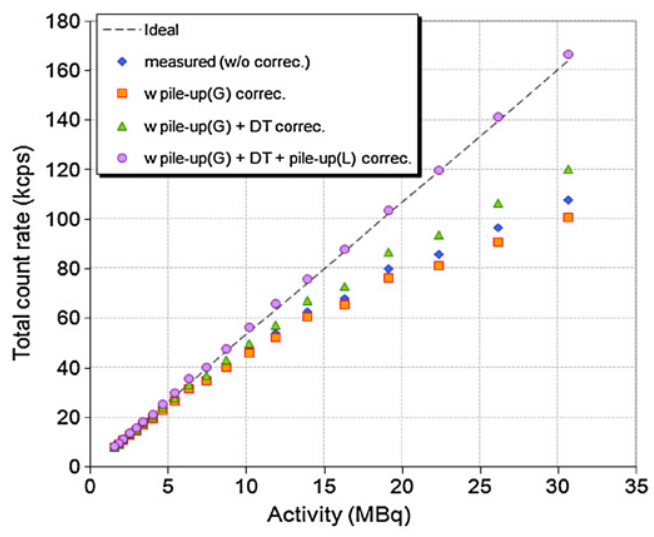

Figure 3. (A) Linear fits used to derive $\tau$ for the Water (SC) phantom using the rPET scanner with an energy window of 400-700 keV. The dead-time correction factor $[m(t)]_{d} /[n(t)]_{d}$ plotted against the measured count rates exhibits an approximate linear behaviour in the range considered. The effective $\tau$ is obtained from the slope. (B) Simulation showing the different contributions to effective dead-time for the case shown in (A), as a function of activity in the FOV.

Besides simulations, experimental data were used to explore the relationship between $\tau$ and SCR for the rPET scanner. The Water (SC) and Water $(L C)$ phantoms were used as calibration acquisitions for the SCR method to calculate the calibration parameters, namely $\tau_{C}$ and $\tau_{S}$, from equation (4). An additional acquisition of a Hot Derenzo phantom was used to compare the $\tau$ value obtained from the decaying source method and that obtained using the computed $\tau_{C}$ and $\tau_{S}$ from equation (4) (SCR method).

\section{Results and discussion}

Figure 3(A) shows the results of the fit to obtain $\tau$ (effective dead-time) values from a decaying source experiment using both experimental data and simulations of the Water (SC) phantom acquired with the rPET scanner. The simulated data show a larger dispersion due to the lower number of counts in each frame, compared to the experimental acquisition. The value obtained by fitting the linear equation to the experimental data (using the non-paralyzable model) is $3.17 \pm 0.04 \mu \mathrm{s}(95 \%$ confidence level), which is comparable to the value of $3.3 \pm 0.1 \mu \mathrm{s}$ obtained from the simulations. In this latter case, we also know the components, which are $2.31 \pm 0.08 \mu$ s from pile-up losses, $0.67 \pm 0.02 \mu$ s from pile-up gains and $1.60 \pm 0.01 \mu \mathrm{s}$ of intrinsic (not pile-up-related) dead-time (see figure 3(B)).

Below, we address the validation of the SCR method proposed in this work for estimating dead-time corrections. First of all, in order to illustrate the dependence of pile-up effects on the SCR, we show in figure 4 the relative contribution (in percentage, left $Y$ axis) of pile-up, dead-time and random counts as a function of the total activity of the acquisition (top $X$ axis) for two simulated acquisitions ((A) and (B)) of the rPET scanner for an energy window of 400-700 keV. The plots also show the dependence of these nonlinear effects on the total detected coincidence rates (shown in the bottom $X$ axis) corresponding to each activity and the total detected singles rates (cross-shaped symbols, right $Y$ axis). Empty symbols in the figure represent coincidence losses; solid ones show detected coincidences.

These two simulations have very different $\operatorname{SCR}\left(\mathrm{SCR}_{\text {Water }(S C)}=19\right.$ (figure $4(\mathrm{~A})$ ), $\mathrm{SCR}_{\mathrm{Rat}(\mathrm{FOV} 2)}=67$ (figure 4(B))). Indeed this can be appreciated in the figure, where both 
(A)

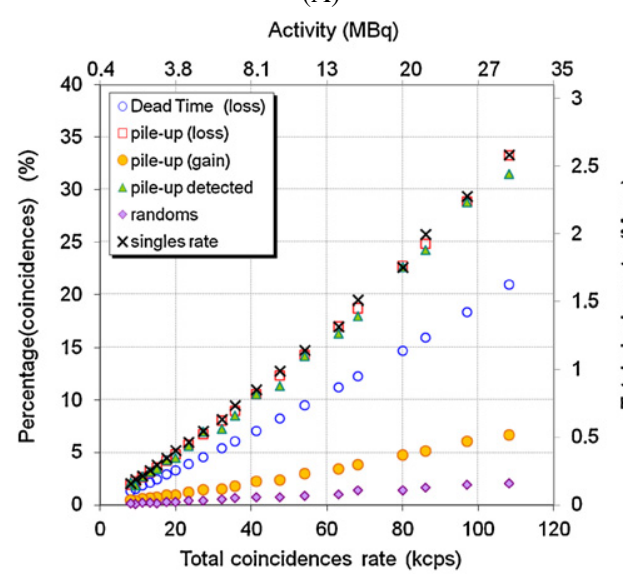

(B)

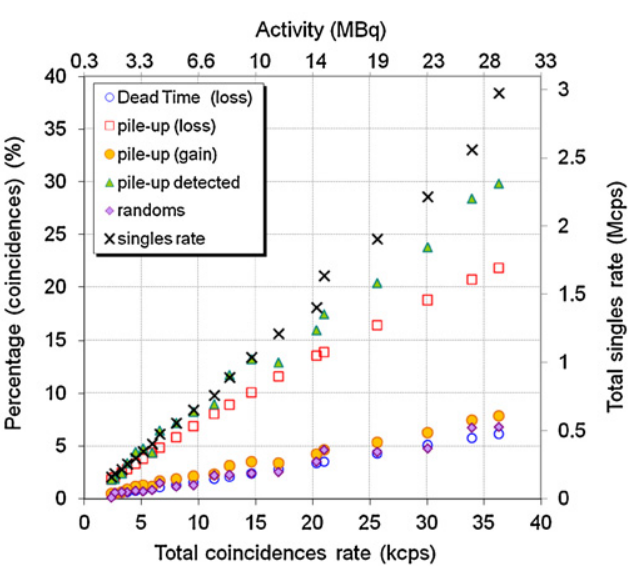

Figure 4. Relative contributions of nonlinear effects to the total coincidence rate (left $Y$ axis) and total singles rate (right $Y$ axis) obtained from simulations of the rPET scanner in an energy window of 400-700 keV. (A) Water (SC) phantom (SCR = 19). (B) Rat (FOV 2) (SCR = 67).

simulations exhibit similar singles rates (cross-shaped symbols, right $Y$ axis) but corresponding to very different coincidence rates (bottom $X$ axis).

We can see that in both cases pile-up is the most significant nonlinear contribution. Pile-up is a limiting factor in this scanner due to the large area of its detectors. In other systems where a smaller solid angle is read by every acquisition channel that processes the detected events, count rates tend to be more limited by the bandwidth of the data acquisition system rather than by the singles rates, and pile-up effects become less important (Mankoff et al 1989, Smith et al 1994, Vicente et al 2007, 2008).

Figure 4 also shows that the behaviour of dead-time losses is approximately independent of the SCR, i.e. both acquisitions present the same percentage of dead-time losses at approximately the same rate of detected coincidences. Nevertheless, pile-up coincidences (losses and gains) become more important as the SCR increases. Furthermore, the behaviour of 'Pile-up detected' (triangle-shaped symbols), which takes into account all the pile-up coincidences detected in the acquisition (including pile-up gains) suggests that not only a count rate linearization is desirable in this case, but also an additional correction of the pile-up coincidences present in the acquisition would be advisable (Vicente et al 2008).

Although coincidence losses and gains due to pile-up do not show the same dependence on the count rate for acquisitions with different SCR, it is possible to obtain an effective deadtime for any acquisition, employing only two calibration acquisitions, one with high SCR and one with low SCR. This procedure works because the effective $\tau$, as it has been shown, has essentially a linear dependence on the SCR.

Table 2 presents the calibration parameters (intercept $\tau_{C}$ and slope $2 \tau_{S}$ ) obtained from rPET scanner simulations for three different energy windows (100-700, 250-700 and 400$600 \mathrm{keV}$ ) and those obtained from the Argus scanner in an energy window of 400-700 keV.

The results of table 2 reveal, for the rPET scanner, a slope almost independent of the energy window, because it depends on the effective dead-time for singles and no energy window was applied in this work to singles measurements. In addition, given the way the rPET system works, the coincidences-only dead-time (intercepts) approaches the conversion time of the ADC of the scanner ( $\tau_{C}=1.6 \mu \mathrm{s}$, see above) for the wider energy window and increases with narrower energy windows. Events that are identified as part of a coincidence are integrated, 
(A)

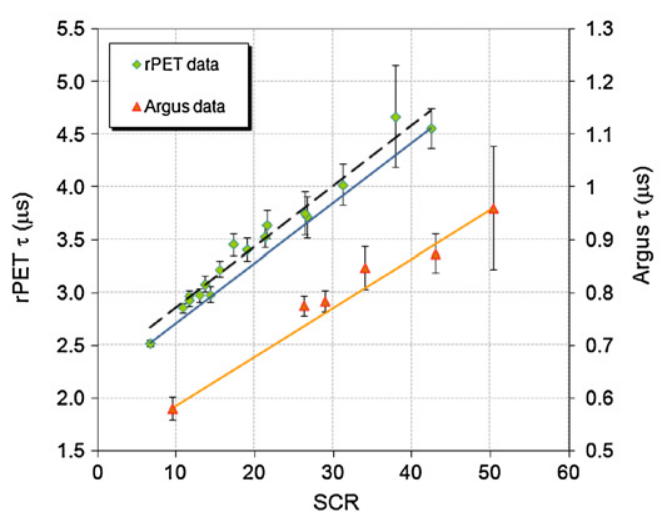

(B)

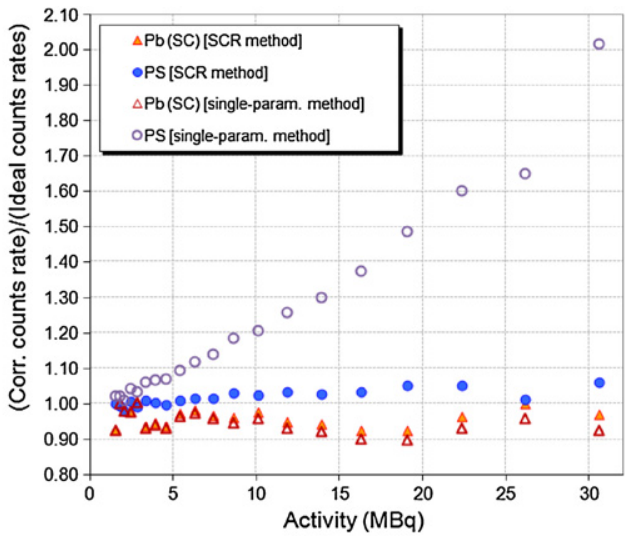

Figure 5. (A) Effective dead-time $\tau$ versus SCR for different acquisitions of the rPET scanner (diamonds, left $Y$ axis) and Argus scanner (triangles, right $Y$ axis) (400-700 keV). The error bars are the estimated errors of the slope $(\tau)$ of each fit using the decaying source method (see figure 3(A)). The dashed line is a linear fit to all rPET points, and the solid line is obtained from the two calibration points of the SCR method at the extreme values of SCR, namely the PS and Water $(L C)$ phantoms. A similar line is also shown for the Argus scanner. (B) Comparison of the ratio of corrected over ideal rates for the SCR method (solid points) and the single-parameter method, which uses only one value for the effective dead-time ('single-param. method', empty points) for the $P S$ and the $P b(S C)$ phantoms. In both cases the SCR method provides more accurate results, with a relative error smaller than $7 \%$ for the $P b(S C)$ phantom and $6 \%$ for the $P S$ one.

Table 2. Linear fit parameters to calculate the effective dead-time $(\tau)$ as a function of SCR, for rPET (three energy windows) and Argus scanners (simulated data).

\begin{tabular}{lllc}
\hline \multirow{2}{*}{ System } & Energy window $(\mathrm{keV})$ & \multicolumn{2}{c}{ Linear fit parameters } \\
\cline { 3 - 4 } rPET & $100-700$ & $1.66 \pm 0.09$ & $0.048 \pm 0.009$ \\
& $250-700$ & $2.05 \pm 0.07$ & $0.046 \pm 0.006$ \\
& $400-700$ & $2.20 \pm 0.09$ & $0.059 \pm 0.008$ \\
Argus & $400-700$ & $0.54 \pm 0.04$ & $0.0089 \pm 0.0008$ \\
\hline
\end{tabular}

converted and further processed. The energy window is applied after conversion. For the narrower energy windows, some events are integrated and subsequently discarded because they fall outside the energy window. Thus, counts that keep the acquisition electronics busy and contribute to dead-time will not remain in the acquired data. The effective coincidence dead-time increases approximately as the inverse of the fraction of counts that survive the energy window. This table also shows that singles dead-time values are relatively small, below $0.03 \mu \mathrm{s}$, both for rPET and Argus. Thus, we can expect equation (4) to be valid, even for singles rates of several Mcps per detector, well above the operating range of both scanners.

Figure 5(A) shows a plot of calculated effective dead-time versus SCR from simulations of the decaying phantoms described in table 1, for the case of rPET (diamonds) and Argus (triangles) scanners in the 400-700 keV energy window. The solid lines represent the relationship between the effective dead-time and SCR estimated using our proposed SCR method with two calibration acquisitions, namely the $P S$ and the Water $(L C)$. These phantoms are representative of the extreme sides of the SCR range. This calibration-estimated line lies slightly below the linear fit using all rPET phantoms (dashed line), but with a similar slope. 
Table 3. SCR and effective dead-time for two bed positions of a voxelized rat simulation (rPET scanner; energy window: 400-700 keV).

\begin{tabular}{lllll}
\hline Simulated study & Description & SCR & Actual $\tau(\mu \mathrm{s})$ & $\tau$ from the SCR method $(\mu \mathrm{s})$ \\
\hline FOV 1 & $\begin{array}{l}\text { Hot bladder at the } \\
\text { edge of the FOV }\end{array}$ & 54 & $4.9 \pm 0.4$ & $5.3 \pm 0.5$ \\
FOV 2 & $\begin{array}{l}\text { Hot bladder outside } \\
\text { the FOV }\end{array}$ & 67 & $5.7 \pm 0.6$ & $6.1 \pm 0.6$ \\
\hline
\end{tabular}

Table 4. SCR (measured at low activity) and effective dead-time from experimental acquisitions of the rPET scanner in the 400-700 keV energy window. Water (SC) and Water (LC) phantoms were used to calibrate (intercept $\tau_{C}$ and slope $2 \tau_{S}$ ) the SCR method.

\begin{tabular}{lccl}
\hline Phantom name & SCR & Actual $\tau(\mu \mathrm{s})$ & $\tau$ from the SCR method $(\mu \mathrm{s})$ \\
\hline Water $(S C)$ & 20 & $3.17 \pm 0.04$ & $\tau=0.053 \cdot \operatorname{SCR}+2.11$ \\
Water $(L C)$ & 43 & $4.4 \pm 0.2$ & \\
\hline Derenzo & 36 & $3.9 \pm 0.2$ & $4.0 \pm 0.3$ \\
\hline
\end{tabular}

The relative error between intercept values is $6 \%$. Nevertheless, the SCR calibration method leads to an error in the corrected count rate smaller than $7 \%$, even with high count rates, for all the phantoms studied. An example of this is shown in figure 5(B).

Figure 5(B) compares the single-parameter method with the method proposed in this work (SCR method) for the rPET scanner in the 400-700 keV energy window. For the singleparameter method, we used the Water $(S C)$ phantom to obtain the fixed $\tau$ value. In the figure, we plot the ratios of corrected over ideal count rates for two situations: a point source (PS) and the small cylinder of lead $(P b(S C))$. The $P S$ was chosen to show the magnitude of the error in the corrected count rates when the effective dead-time is overestimated, as happens when using the dead-time obtained with the single-parameter method from the Water (SC) to correct the count rates of the $P S$ acquisition. The second example, $P b(S C)$, was selected because this point has the worst fit to the calibration-estimated line for the rPET scanner with our SCR method (see figure 5(A), point: $(37,4.7)$ ). We can see that in both cases the SCR method provides more accurate results, with a relative error smaller than $7 \%$ for the $P b(S C)$ phantom and less than $6 \%$ for the $P S$, compared to deviations of $10 \%$ for the $P b(S C)$ phantom and even up to $100 \%(P S)$ when the single-parameter correction is used. This example shows that the single-parameter correction introduces a bias in the corrected count rates. The error is large if the $\tau$ used for the correction overestimates the actual $\tau$ value of the acquisition and will be more significant for large detector blocks; therefore, the SCR method would be highly recommended for clinical dedicated breast PET scanners (or PEM cameras), as for example those described in Wang et al (2006a) and Wu et al (2009), or for high-sensitivity preclinical PET scanners with particularly close detectors, such as those described in Seidel et al (2010) and Zhang et al (2011).

In table 3 we quote SCR and effective $\tau$ values for two different bed positions of a rat simulation. The effective $\tau$ calculated using the decaying source method, which we consider the actual value (column 4), is compared with the one obtained using the SCR method (column 5). Differences between both values are smaller than $8 \%$ (within error bars) for both cases. This example confirms that the proposed method works fine even when the activity distribution is not symmetric along the axial or transaxial FOV.

Besides simulations, the relationship between $\tau$ and SCR for the rPET scanner was also explored with experimental data. Table 4 shows the parameters $\tau$ (calculated using the decaying source method, column 3) and SCR from the Water (SC) and the Water $(L C)$ phantoms in the 
400-700 keV energy window, which are the two acquisitions used in the calibration phase of the SCR method. The effective $\tau$ obtained from the Derenzo decaying experiment, which we consider the actual value (column 3), and the one estimated from the SCR method (column 4) differ by about $3 \%$. In addition, the experimental values of the effective dead-time for these rPET acquisitions are within $8 \%$ of those of the simulations (table 2).

\section{Conclusions}

This paper proposes a method to calculate for each individual acquisition the effective deadtime $\tau$ that allows us to restore the correct linear relationship between activity in the FOV and count rate measured by the scanner, thus removing nonlinear effects in the count rate due to dead-time and pile-up losses and pile-up gains. The method addresses the fact that each acquisition exhibits a different effective $\tau$, since pile-up effects depend on the material, object size and source distribution inside and outside the FOV. It requires only two calibration acquisitions and is based on the linear relationship between $\tau$ and SCR.

In contrast, the standard procedure that uses a single value for effective dead-time $\tau$ to correct any acquisition (Knoll 2000, Germano and Hoffman 1988) introduces a bias in the corrected count rates, especially in situations when the effective $\tau$ of the correction overestimates the actual value $\tau$ of the acquisition, with errors sometimes up to $100 \%$ for high activities.

We used simulated and experimental data to verify that the SCR method performs well for two commercial preclinical PET scanners with very different architectures: rPET (Vaquero et al 2005), a small-animal PET scanner with four rotating large-area detectors without pile-up rejection in the electronics and for which pile-up is relatively important, and the Argus scanner (Wang et al 2006b), a small-animal PET scanner with more conventional geometry (two full rings) and small-area detectors. Simulations show that the error in the corrected count rates is below 7\%, even when high activities are present in the FOV. Even smaller errors could be attained using more than two phantoms to calibrate the method.

The SCR method could be applied in any other scanner, including clinical ones, and it will be of higher interest in scanners with large detector blocks, such as clinical dedicated breast PET scanners (or PEM cameras) (Wang et al 2006a, Wu et al 2009) or high-sensitivity preclinical PET scanners with close-up detectors (Seidel et al 2010, Zhang et al 2011).

\section{Acknowledgments}

This work was partially funded by AMIT project (CEN-20101014) from the CDTICENIT program, CIBERsam (CB07/09/0031), projects TEC2010-21619-C04-01 and TEC2011-28972-C02-01 from Spanish Ministerio de Ciencia e Innovación, Spanish Government (ENTEPRASE Grant, PSE-300000-2009-5), PRECISION grant IPT-3000002010-3, CPAN (CSD-2007-00042@Ingenio2010), MEC (FPA2010-17142) and ARTEMIS program (S2009/DPI-1802) from Spanish Comunidad de Madrid and EU-ERDF program. Part of the calculations of this work were performed in the 'Clúster de Cálculo de Alta Capacidad para Técnicas Físicas' funded in part by UCM and in part by UE with European regional funds. This is a contribution from the Moncloa Campus of International Excellence.

\section{References}

Badawi R D and Marsden P K 1999 Self-normalization of emission data in 3D PET IEEE Trans. Nucl. Sci. 46 709-12 Badawi R D, Miller M P, Bailey D L and Marsden P K 1999 Randoms variance reduction in 3D PET Phys. Med. Biol. 44 941-54 
Bendriem B and Townsend D W 1998 The Theory and Practice of 3D PET (Dordrecht: Kluwer)

Brasse D, Kinahan PE, Lartizien C, Comtat C, Casey M and Michel C 2005 Correction methods for random coincidences in fully $3 \mathrm{D}$ whole-body PET: impact on data and image quality J. Nucl. Med. 46 859-67 (PMID: 15872361)

Casey ME and Hoffman E J 1986 Quantitation in positron emission computed tomography: 7. A technique to reduce noise in accidental coincidence measurements and coincidence efficiency calibration J. Comput. Assist. Tomogr. 10 845-50

Cherry S R, Sorenson J A and Phelps M E 2003 Physics in Nuclear Medicine 3rd edn (Philadelphia, PA: Saunders)

Daube-Witherspoon ME and Carson R E 1991 Unified deadtime correction model for PET IEEE Trans. Med. Imaging 10 267-75

Eriksson L, Wienhard K and Dahlbom M 1994 A simple data loss model for positron camera systems IEEE Trans. Nucl. Sci. 41 1566-70

España S, Herraiz J L, Vicente E, Vaquero J J, Desco M and Udias J M 2009 PeneloPET, a Monte Carlo PET simulation tool based on PENELOPE: features and validation Phys. Med. Biol. 54 1723-42

Furrer M, Gostely J J and Lerch P 1984 Timing problems due to pile-up in coincidence counting Nucl. Instrum. Methods Phys. Res. A 226 455-62

Germano G and Hoffman E J 1988 Investigation of count rate and deadtime characteristics of a high resolution PET system J. Comput. Assist. Tomogr. 12 836-46

Germano G and Hoffman E J 1990 A study of data loss and mispositioning due to pileup in 2-D detectors in PET IEEE Trans. Nucl. Sci. 37 671-75

Germano G and Hoffman E J 1991 An investigation of methods of pileup rejection for 2D array detectors employed in high resolution PET IEEE Trans. Med. Imaging $10223-7$

Hoffman E J, Phelps M E and Huang S C 1983 Performance evaluation of a positron tomograph designed for brain imaging J. Nucl. Med. 24 245-57 (PMID: 6600782)

Imperiale C and Imperiale A 2001 On nuclear spectrometry pulses digital shaping and processing Measurement 30 49-73

Knoll G F 2000 Radiation Detection and Measurement (New York: Wiley)

Mankoff D A, Muehllehner G and Karp J S 1989 The high count rate performance of a two-dimensionally positionsensitive detector for positron emission tomography Phys. Med. Biol. 34 437-56

Mazoyer B M, Roos M S and Huesman R H 1985 Dead time correction and counting statistics for positron tomography Phys. Med. Biol. 30 385-99

Seidel J, Wenze X, Kakareka J W, Pohida T J, Green M V and Choyke P L 2010 A positron projection imager for whole-body mouse imaging 2010 IEEE Nuclear Science Symp. Conf. Record (NSS/MIC) (30 October-6 November 2010) pp 2206-9

Sjöland K A and Kristiansson P 1994 Pile-up and defective pulse rejection by pulse shape discrimination in surface barrier detectors Nucl. Instrum. Methods Phys. Res. B 94 333-7

Smith R J, Karp J S and Muehllehner G 1994 The countrate performance of the volume imaging PENN-PET scanner IEEE Trans. Med. Imaging 13 610-8

Stearns C W, Chesler D A, Kirsch J E and Brownell G L 1985 Quantitative imaging with the MGH analog ring positron tomograph IEEE Trans. Nucl. Sci. 32 898-901

Stearns C W, McDaniel D L, Kohlmyer S G, Arul P R, Geiser B P and Shanmugam V 2003 Random coincidence estimation from single event rates on the discovery ST PET/CT scanner 2003 IEEE Nuclear Science Symp. Conf. Record (19-25 October 2003) vol 5 pp 3067-9

Thompson C J and Meyer E 1987 The effect of live time in components of a positron tomograph on image quantification IEEE Trans. Nucl. Sci. 34 337-43

Vaquero J J, Lage E, Ricon L, Abella M, Vicente E and Desco M 2005 rPET detectors design and data processing 2005 IEEE Nuclear Science Symp. Conf. Record (23-29 October 2005) pp 2885-9

Vicente E, Espana S, Lopez Herraiz J, Herranz E, Desco M, Vaquero J J and Udias J M 2008 Nonlinear effect of pile-up in the quantification of a small animal PET scanner IEEE Nuclear Science Symp. Conf. Record, NSS'08 (19-25 October 2008) pp 5391-5

Vicente E, Soto-Montenegro M, Espana S, Herraiz J L, Herranz E, Vaquero J J, Desco M and Udias J M 2007 Influence of random, pile-up and scatter corrections in the quantification properties of small-animal PET scanners IEEE Nuclear Science Symp. Conf. Record, NSS'07 (26 October-3 November 2007) pp 3964-8

Wang G C, Huber J S, Moses W W, Qi J and Choong W S 2006a Characterization of the LBNL PEM camera IEEE Trans. Nucl. Sci. 53 1129-35

Wang Y, Seidel J, Tsui B M, Vaquero J J and Pomper M G 2006b Performance evaluation of the GE healthcare eXplore VISTA dual-ring small-animal PET scanner J. Nucl. Med. 47 1891-900 (PMID: 17079824)

Williams C W, Crabtree M C and Burgiss S G 1979 Design and performance characteristics of a positron emission computed axial tomograph-ECATÂ ${ }^{\mathbb{R}}$-II IEEE Trans. Nucl. Sci. 26 619-27 
Wu Y, Bowen S L, Yang K, Packard N, Fu L, Burkett G, Qi J, Boone J M, Cherry S R and Badawi R D 2009 PET characteristics of a dedicated breast PET/CT scanner prototype Phys. Med. Biol. 54 4273-87

Yamamoto S, Amano M, Miura S, Iida H and Kanno I 1986 Deadtime correction method using random coincidence for PET J. Nucl. Med. 27 1925-8 (PMID: 3491196)

Yu D F and Fessler J A 2000 Mean and variance of single photon counting with deadtime Phys. Med. Biol. 452043

Zhang H, Bao Q, Vu N T, Silverman R W, Taschereau R, Berry-Pusey B N, Douraghy A, Rannou F R, Stout D B and Chatziioannou A F 2011 Performance evaluation of PETbox: a low cost bench top preclinical PET scanner Mol. Imaging Biol. 13 949-61 